\title{
TRANSACTIONS MONÉTAIRES EN PAYS KANAK
}

\author{
Elsa Faugère
}

Belin | Genèses

$2000 / 4$ - no 41

pages 41 à 62

ISSN 1155-3219

Article disponible en ligne à l'adresse:

http://www.cairn.info/revue-geneses-2000-4-page-41.htm

Pour citer cet article :

Faugère Elsa , «Transactions monétaires en pays Kanak »,

Genèses, $2000 / 4$ no 41 , p. 41-62.

Distribution électronique Cairn.info pour Belin.

(c) Belin. Tous droits réservés pour tous pays.

La reproduction ou représentation de cet article, notamment par photocopie, n'est autorisée que dans les limites des conditions générales d'utilisation du site ou, le cas échéant, des conditions générales de la licence souscrite par votre établissement. Toute autre reproduction ou représentation, en tout ou partie, sous quelque forme et de quelque manière que ce soit, est interdite sauf accord préalable et écrit de l'éditeur, en dehors des cas prévus par la législation en vigueur en France. II est précisé que son stockage dans une base de données est également interdit. 


\section{TRANSACTIONS}

\section{MONÉTAIRES}

EN PAYS KANAK*

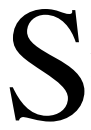
elon une représentation communément admise en Nouvelle-Calédonie, les tribus kanakes seraient restées à l'écart de l'économie monétaire et marchande néo-calédonienne. La responsabilité de cette mise à l'écart est renvoyée du côté de la coutume et donc des Kanaks. S'ils sont marginalisés, dit-on, c'est à cause de leur coutume qui s'oppose à l'argent et au marché. Euxmêmes partagent d'ailleurs un tel point de vue. Pourtant, cette représentation dominante est en décalage avec leurs pratiques effectives, tout au moins avec celles des Kanaks de l'île de Maré où j'ai fait mes enquêtes de terrain. À Maré, de nombreux gestes coutumiers sont monétaires. Et, les Maréens participent pleinement à l'économie marchande. Ceci dit, la monétarisation de leurs coutumes pose un problème aux Maréens et la marchandisation de certaines de leurs transactions se fait avec difficultés. Si leur coutume est bel et bien monétarisée, ils considèrent que ce n'est plus la «vraie coutume» et que cette monétarisation menace leur ordre social. Une telle vision est due à une représentation très occidentale de l'argent qui le dote du pouvoir intrinsèque de révolutionner la société et la culture. Cette diabolisation de l'argent révèle en fait une erreur très répandue qui consiste à confondre argent et marché.

Le terme de «coutume», abondamment utilisé par les Maréens, est la traduction française du terme nengone ${ }^{1}$ «penenod». Littéralement, ce terme signifie: «la manière de faire au pays ${ }^{2}$.» Le terme «coutume» est utilisé à différents niveaux: pour désigner l'ensemble de ce qui est considéré comme «tradition» kanake. On dira alors «c'est la coutume». "Coutume» sert aussi à désigner une cérémonie particulière: mariage, deuil, fête des ignames, etc. On dira alors «c'est une coutume». Quant à la formule «faire la coutume», elle qualifie un ou plusieurs
* Je remercie chaleureusement Florence Weber, Isabelle Merle et Michel Naepels pour leurs lectures attentives, critiques et constructives des versions antérieures de cet article.

1. Nom de la langue vernaculaire de l'île de Maré.

2. Pene, c'est-à-dire manière de; nod, c'est-à-dire pays. 


\section{O S S I E R}

Comment décrire les transactions Elsa Faugère Transactions monétaires en pays kanak
3. 1 FCFP (Franc Pacifique) correspond à $0,055 \mathrm{FF}$, donc $100 \mathrm{FCFP}$ à $5,5 \mathrm{FF}$. gestes qui s'effectuent à certaines occasions, notamment dans les cérémonies familiales; ou encore, quand on donne une petite somme d'argent en arrivant chez quelqu'un que l'on n'a pas vu depuis longtemps: en lui versant $100 \mathrm{FCFP}^{3}$, «on lui fait la coutume».

La description et l'analyse d'une demande en mariage montreront que l'utilisation de l'argent dans cette cérémonie ne la transforme pas pour autant en halle marchande. L'argent y sert à refléter et à entretenir les liens de parenté et à en créer de nouveaux avec la parenté de la future mariée. Il y existe une étiquette des dons d'argent qui permet de bien les distinguer d'autres types de transferts monétaires. $\mathrm{Si}$, au cours de la cérémonie, l'argent qui circule n'est pas un moyen de paiement, en dehors il peut en être un. De là provient la crainte des Maréens. Cette société très hiérarchisée et inégalitaire où les positions sociales sont héréditaires se trouve confrontée à d'autres modalités de valorisation sociale et d'acquisition du prestige. Dénigrer l'argent et ceux qui en gagnent et valoriser tout ce qui relève de la coutume peut s'interpréter comme une forme de résistance aux mutations sociales et économiques liées à la politique de rééquilibrage mise en œuvre, depuis 1988, par les accords de Matignon. Cette valorisation de la coutume permet finalement de préserver une certaine égalité matérielle en mettant la pression sur ceux qui «sortent du lot», c'est-à-dire sur ceux qui gagnent de l'argent. Cette pression se manifeste et s'exprime au travers d'incessantes rumeurs de jalousie susceptibles de donner lieu à des attaques en sorcellerie. Une menace plane en permanence sur ceux qui «réussiraient» trop bien ou qui le montreraient trop: ils risqueraient ainsi de provoquer la jalousie des autres qui ne manqueraient pas de leur jeter de mauvais sorts. Ceux qui auraient des velléités d'afficher leur réussite matérielle sont donc confrontés à ce risque de jalousie et de sorcellerie ou plutôt à ces rumeurs de risque. Mais, on le verra, cette menace peut toutefois être écartée en se convertissant au pentecôtisme.

Aussi existe-t-il un deuxième risque, ou plutôt une deuxième rumeur de risque: le «profitage». Les incessantes accusations de «profitage» servent finalement, elles aussi, à maintenir une certaine égalité matérielle (on préfère ne pas montrer sa réussite pour ne pas courir le risque d'en redistribuer une partie) et à freiner, en les stigmatisant, les velléités de ceux qui chercheraient vraiment à «profiter» des autres. Il est tellement difficile voire impossible de refuser quelque chose à un parent, 
comme le montrera le cas d'Albert, qu'il vaut mieux stigmatiser le fait de demander. Dans cette société d'interconnaissance où tout le monde est plus ou moins apparenté, cette stigmatisation permet de réguler les échanges et de les contrôler. C'est un moyen pour éviter une forme d'anarchie transactionnelle où l'on pourrait demander tout et n'importe quoi à n'importe qui, n'importe où et n'importe quand. Au contraire, les transactions sont très codifiées et réglementées. L'un des moyens utilisés localement pour permettre l'instauration de transactions marchandes entre parents est le recours à un contrôle collectif, comme dans l'espace du marché municipal.

Dans cet article, j'analyse les difficultés rencontrées par les Maréens lorsqu'ils effectuent deux types de transactions: monétaires et non marchandes, d'un côté, monétaires et marchandes, de l'autre. Dans une première partie, je m'interroge sur les raisons pour lesquelles les Maréens critiquent et craignent la monétarisation de leurs coutumes (transactions monétaires non marchandes), en analysant celles-ci à travers le cas d'une cérémonie de fiançailles. Si les Maréens sont effectivement insérés dans une économie de marché, les transactions monétaires et marchandes au sein de la parenté leur posent, dans certaines situations, des problèmes. L'analyse de ces difficultés est l'objet de la deuxième partie de l'article.

\section{L'argent dans les cérémonies coutumières}

L'argent occupe une place tout à fait singulière dans la société néo-calédonienne. Il sert à entretenir les «frontières ethniques», pour reprendre la terminologie de Frederic Barth ${ }^{4}$. Les discours tant des métropolitains que des Kanaks ou des Caldoches se plaisent à opposer la coutume à l'argent: «les Kanaks c'est la coutume et les Européens c'est l'argent». Cette opposition discursive n'est pas surprenante en elle-même. Ce qui l'est davantage c'est qu'elle est en contradiction avec les pratiques effectives des Maréens. Ils utilisent en effet l'argent de manière quasi systématique pour «faire leurs coutumes» et souvent en quantité importante, tout en regardant cette monétarisation des pratiques coutumières comme un danger et une menace pour leur ordre social, et comme le signe de la déliquescence et du travestissement de la «vraie coutume». Ils vont jusqu'à se poser la question suivante: comment peut-on être et rester Maréen en ayant une coutume aussi monétarisée?
4. Frederic Barth (éd.),

Ethnic Groups and Boundaries. The social organization of culture difference, Bergen-Oslo, Universitets Forlaget, Londres, Georges Allen et Unwin, 1969. 


\section{O S S I E R}

Comment décrire les transactions Elsa Faugère Transactions monétaires en pays kanak
5. Jonathan Parry, Maurice Bloch (éd.), Money and the Morality of Exchange, Cambridge, Cambridge University Press, 1989, introduction, pp. 2 et suiv. Toutes les traductions françaises des textes anglais sont de moi.

6. Ibid.

7. Viviane A. Zelizer, The Social Meaning of Money, New York, Basic Books, 1994, pp. 6, 10 et suiv.

8. Ibid., p. 4.

9. J. Parry et M. Bloch, Money and the Morality..., op. cit., pp. 4, 7, 8.
Les craintes des Maréens, leur inquiétude et leur peur que l'argent ne perturbe irrémédiablement l'ordre social ressemblent à s'y méprendre à certaines théories occidentales sur l'argent. Le discours occidental sur l'argent se caractérise par deux tendances opposées: l'une, issue d'Aristote, condamne l'argent et le commerce, les estimant contre nature et destructeurs des liens sociaux. L'autre tendance, issue d'Adam Smith, considère, comme le soulignent Jonathan Parry et Maurice Blochs, que le bonheur et la prospérité d'une société sont basés sur la recherche individuelle du profit monétaire Ces deux auteurs soutiennent, de façon convaincante, que ces différents courants de notre tradition culturelle partent en fait d'un même point de vue selon lequel l'argent serait doté d'un pouvoir intrinsèque de révolutionner la société et la culturé.

Faisant un historique des théories occidentales sur l'argent, Viviane A. Zelizer aboutit au même constat: "le pouvoir de l'argent de transformer la société moderne s'est emparé de l'imagination des théoriciens sociaux du XIX et du début du XX ${ }^{\mathrm{e}}$ siècle.»Et, ajoute-telle, «les sociologues contemporains continuent d'adhérer à l'idée que l'argent est un moyen d'échange marchand, parfaitement fongible, neutre qualitativement, indéfiniment divisible et entièrement homogène. Ainsi, les sociologues continuent d'accepter, avec une absence remarquable de scepticisme, l'idée que dès que l'argent a envahi le domaine des relations personnelles, il conduit inévitablement ces relations vers une rationalité instrumentale ${ }^{7}$. $\gg$ Alors qu'ils ont depuis longtemps admis le temps social et l'espace social, l'argent social leur a échappé ${ }^{8}$. Selon cet auteur, nous ne savons quasiment rien de la vie sociale de l'argent. Les théoriciens sociaux qui le considèrent pourtant comme un élément fondamental des sociétés modernes, ne l'analysent pas comme une catégorie sociologique; l'argent n'est pas considéré comme une réalité sociale.

J. Parry et M. Bloch notent que pour Karl Marx, comme pour Georg Simmel, «l'argent est associé à, et promeut, la montée de l'individualisme et la destruction des solidarités communautaires», et que, par la suite, on a sous-estimé voire nié l'importance de la monnaie et des échanges marchands dans de nombreuses économies «traditionnelles» pré-capitalistes. Or, remarquent-ils, «l'augmentation de la monétarisation n'est pas un indice fiable de l'atrophie de "l'économie morale" "». Allant 
dans le même sens, Peter Geschiere montre que les rituels de la parenté des Maka du Cameroun, à l'occasion desquels ont lieu de féroces marchandages monétaires, vont à l'encontre de la dichotomie occidentale entre la sphère de la parenté et celle de l'argent:

«Apparemment, si j'ai été abasourdi par le rôle ostensible de l'argent dans les rituels de la parenté chez les Maka, c'est parce que ce rôle s'accordait mal avec certaines distinctions fondamentales dans la pensée occidentale:

- la distinction, au moins formelle, entre la sphère de la parenté et celle de l'argent et du marché;

- la distinction entre les sociétés dominées par le "marché", dont l'Occident est censé être le meilleur exemple, et les sociétés dans lesquelles le "marché" (ou "l'économie") est encore enclavé et bridé par des principes socioculturels différents ${ }^{10}$.»

Mon étonnement devant la forte monétarisation des travaux coutumiers maréens émanait très probablement de cette même idéologie occidentale évoquée par P. Geschiere. Le choix de prendre comme thématique de recherche «l'argent et la parenté» signifiait que je percevais, dans l'imbrication effective de ces deux termes à Maré, une relation problématique. Pour paraphraser P. Geschiere ${ }^{11}$, je dirai que face à la tendance occidentale à distinguer les sphères de la parenté et celle de l'argent et du marché, l'exemple maréen surprend par l'aisance avec laquelle les «rituels de la parenté » se sont associés à la monétarisation. Cependant, contrairement aux Maka du Cameroun, la monétarisation effective des «rituels de parenté » est l'objet de vives critiques de la part des Maréens. Ces critiques peuvent être interprétées comme le signe d'une appropriation de l'idéologie occidentale selon laquelle «argent» et «parenté» doivent rester dissociés. Ce serait alors, apparemment, le comble du paradoxe puisque les Maréens légitiment ces critiques par le rejet de cette même idéologie occidentale et de la présence coloniale. Mais le paradoxe n'est qu'apparent. En effet, si des logiques identitaires et politiques sous-tendent effectivement ces résistances discursives à la monétarisation des travaux coutumiers, de nombreux auteurs ont montré que les processus de construction des identités «ethniques » apparaissent lorsqu'il existe une implication effective dans les activités et les rôles de la société globale:

«Contrairement aux hypothèses des théories assimilationnistes, ce n'est pas le repliement sur soi et l'isolement, mais au contraire l'implication dans les activités et les rôles de la société globale qui rendent saillante la conscience ethnique.
10. Peter Geschiere,

«Parenté et argent dans une société lignagère », in Jean-François Bayart (éd.), La réinvention du capitalisme, Paris, Karthala, 1994, pp. 88 et suiv.

11. Ibid., p. 91. 


\section{O S S I E R}

Comment décrire les transactions Elsa Faugère

Transactions monétaires en pays kanak
12. Philippe Poutignat et Jocelyne Streiff-Fenart,

Théories de l'ethnicité, Paris, Puf, 1995, pp. 77 et suiv.

13. Comme le souligne à juste titre Florence Weber dans ce même numéro de Genèses ( «Transactions marchandes, échanges rituels, relations personnelles. Une ethnographie économique après le Grand Partage », $n^{\circ}$ 41, 2000), il y a des transactions marchandes qui ne sont pas monétaires (le troc) et des transactions monétaires qui ne sont pas marchandes (les travaux coutumiers des Maréens par exemple).
[...] L'acquisition des compétences et des valeurs modernes reste le processus central de la transformation des identités ethniques, mais loin de conduire à l'assimilation, elle a pour effet d'accroître la conscience et la signification de l'ethnicité ${ }^{12}$.»

Ce qui apparaît en filigrane dans les écrits des différents auteurs que je viens de citer, comme dans les discours des Maréens, c'est la confusion entre argent et marché. L'erreur qui consiste à prendre l'argent (médium de la transaction) pour le marché (nature, type de la transaction) est une erreur très répandue tant dans le sens commun que dans les écrits scientifiques. Monétarisation ne signifie pas marchandisation ${ }^{13}$. À travers l'étude de cas d'un travail coutumier - une demande en mariage - je vais montrer comment la monétarisation de cette cérémonie ne la transforme pas pour autant en un espace marchand.

\section{Étude de cas: Riri attache Élia}

Dans la nuit du 3 février 1996, un jeune homme, Riri, accompagné de sa parenté, part demander sa main à une jeune fille, Élia. Riri et Élia vivent ensemble à Nouméa depuis plusieurs mois. Mais ils sont rentrés pour l'occasion dans leurs tribus respectives. Aussi Élia est informée de la visite qui l'attend cette nuit. La demande en mariage s'effectue toujours en pleine nuit ce qui donne à cette cérémonie une atmosphère très particulière. La première phase de la cérémonie débute chez Kuma, le père de Riri. J'y arrive en début de soirée accompagnée du beau-frère d'un Grand Chef de Maré. Dès notre arrivée, cet homme donne 200 FCFP (11 FF) à Kuma comme «coutume de rentrée». Ce geste coutumier s'appelle le shudrul waruma. Littéralement, cela veut dire "avoir la vie sauve». C'est un geste d'humilité que l'on accomplit toujours avant d'entrer chez un inconnu, chez quelqu'un que l'on n'a pas vu depuis longtemps ou lorsque l'on arrive pour une cérémonie coutumière. Chaque fois qu'il reçoit un don monétaire, Kuma remercie le geste après avoir montré l'argent à tout le monde. Au geste du beau-frère du Grand Chef, il dit: «Je m'abaisse devant vous, je suis jeune, j'ai peur de mon oncle maternel, j'ai peur du Grand Chef, j'ai peur de mon père, j'ai peur de ma mère, j'ai peur de ma sœur.» Kuma nous invite ensuite à entrer chez lui et à nous restaurer.

En plus de ce geste de «coutume de rentrée», certains donnent une somme d'argent bien plus importante pour aider au travail coutumier qui suivra le repas. Le montant 
de chacun de ces dons monétaires n'est pas rendu public. Ce n'est donc qu'après le repas, vers vingt-trois heures, que le travail coutumier proprement dit commence. Près d'un groupe de vieilles femmes jouant par terre aux dominos, se réunissent le père du marié, son oncle maternel, le Grand Chef d'un district voisin et deux hommes de son clan. L'un d'entre eux joue le rôle de «secrétaire» du travail. Dans tout travail coutumier, quelqu'un est chargé d'inscrire sur un cahier le montant et la composition des dons de chacun. Une stricte comptabilité est toujours tenue. L'argent est alors compté dans une indifférence quasi générale. Quelques personnes viennent discrètement donner quelques billets supplémentaires (co enono). Tout se passe en silence, dans la pénombre, presque en cachette. L'ambiance est feutrée. À un certain moment, le père de Riri se lève et montre à tous une somme d'argent. C'est l'argent qui reste d'une première demande en mariage auprès d'une autre femme: après avoir accepté, l'année précédente, de devenir l'épouse de Richard et avoir donc pris l'argent de cette "coutume», elle avait changé d'avis quelques mois plus tard. Le père de cette femme aurait alors dû rendre la totalité de l'argent reçu. Mais il n'en a rendu que la moitié.

Le décompte achevé, Kuma dit au Grand Chef du district voisin: «il y a 331000 FCFP (18205 FF). Donc on donne 10000 (550 FF) pour le dure hnara, 1000 pour le shudrul waruma (coutume de rentrée) et 320000 $(17600 \mathrm{FF})$ pour ataceni». Le Grand Chef acquiesce. Le dure hnara est le geste que l'on adresse à la future épouse pour la remercier d'avoir accepté la demande en mariage. La somme appelée ataceni, qui signifie «attacher», est destinée aux parents (père et mère) de la jeune fille qui a accepté la demande. Une fois l'accord réalisé, le père du marié annonce à l'assistance l'origine du montant total: «224000 proviennent de notre clan et 107000 de votre participation à vous tous». Ensuite, un autre homme annonce la répartition de ces dons: 10000 pour l'épouse, 1000 pour la «coutume de rentrée» et 315000 pour les parents. Les 5000 francs de différence entre l'annonce publique et le décompte réel correspondent à la somme donnée en secret aux hnakasese du futur marié. Ce terme (littéralement «avoir mangé ensemble») désigne une relation clanique considérée comme sacrée. Tout un ensemble de droits et d'interdits caractérisent la relation entre deux clans hnakasese. Les raisons d'une telle relation demeurent mystérieuses ${ }^{14}$.
14. J'ai rendu compte de différentes interprétations de cette relation dans ma thèse: «L'argent et la coutume, Maré, Nouvelle-Calédonie», Paris, EHESS, 1998, pp. 205 et suiv. 


\section{O S S I E R}

Comment décrire les transactions Elsa Faugère

Transactions monétaires en pays kanak
Le travail terminé, Kuma prend avec précaution les liasses de billet, comme s'il tenait entre ses mains un objet précieux, et réalise trois beaux rouleaux qu'il donne à l'oncle maternel de son fils. C'est à lui maintenant d'aller demander la main de la jeune femme. Normalement, quand on fait la répartition de l'argent, un don supplémentaire à ceux que je viens d'évoquer, est réservé à l'oncle maternel du futur marié. Mais dans le cas décrit ici, le don à l'oncle maternel ayant été fait l'année précédente, lors de la demande en mariage finalement non aboutie, il a été considéré qu'il n'était pas à refaire cette fois-ci. Vers minuit, un convoi de voitures se met en route. Il débarque chez les parents d'Élia où personne ne semble prêter attention à cette invasion nocturne. Peu à peu, l'atmosphère devient tendue. L'enjeu est important. Une fois toutes les voitures arrivées, Kuma nous dit de nous approcher. Il donne alors le shudrul waruma au père de la fille qui nous invite à nous asseoir dehors, autour d'une grande table. Mais la foule est trop nombreuse. Il nous fait alors entrer dans la case où chacun s'assoit en silence. Visiblement très ému, il remercie la «coutume de rentrée», en déclarant: «Je m'abaisse devant vous, j'ai peur, j'ai peur parce que je suis jeune, j'ai peur des vieux, j'ai peur du Grand Chef, je ne suis qu'un sujet, un serviteur, c'est la première fois qu'un Grand Chef vient chez moi, j'ai peur, je vous remercie.»

L'oncle maternel de Riri déclare alors que nous sommes venus parler à sa fille, Élia. Son père l'appelle. Il n'obtient pas de réponse. Il quitte la case et revient quelques minutes après, accompagnée de sa fille très émue et impressionnée à la vue de cette case noire de monde. L'oncle maternel lui demande alors si elle veut bien être la femme de Riri. Elle n'a qu'à dire oui ou non. La tension est alors à son comble. Dans un silence de mort, la jeune femme articule faiblement oui. Un immense soulagement se fait alors sentir, et un grand murmure de remerciement brise enfin le silence. Puis l'oncle maternel du marié lui dit: "pour vous remercier de votre oui, voilà 10000 francs. Terminé.» Un vieux lui chuchote alors quelques mots à l'oreille. L'oncle s'excuse et déclare, sortant un beau rouleau de billets de son porte-monnaie: "voilà l'argent pour vos parents, pour votre père et votre mère, pour vous "attacher" ». Il lui tend l'argent et lui dit: "voilà $315000 \mathrm{~F}$ pour vous "attacher" ». La jeune femme prend les billets. Elle les donne à son père aux côtés de sa mère, tous deux en pleurs. 
D'autres discrets sanglots se font entendre dans l'assistance. Le père, puis la mère, terriblement émus, remercient longuement pour ce geste. Ensuite, la mère d'Élia nous invite à prendre un thé autour d'une table bien garnie qui nous attendait dehors.

\section{Techniques de distinction entre dons d'argent et transac- tions monétaires marchandes}

Le rôle joué par l'argent dans le travail coutumier que je viens d'illustrer est majeur. À chaque étape de la cérémonie, les dons d'argent ont un sens particulier. Geste d'humilité pour la coutume de rentrée; geste de solidarité lorsque la parenté du futur marié donne quelques billets au père de celui-ci; geste de pardon et de respect envers l'oncle maternel du marié; geste de remerciement à la future mariée lorsqu'elle accepte le mariage; geste pour attacher cette femme lorsque l'on donne de l'argent à son père et à sa mère. L'argent sert donc dans ce cadre à entretenir des liens sociaux et à en créer de nouveaux. Loin d'être une menace pour leur coutume, comme les Maréens se plaisent à le dire, il en est le principal médium. Il sert à «faire la coutume», à la re-fabriquer par le biais de ces multiples échanges. Ainsi, loin de détruire le tissu social, l'argent apparaît, tout au contraire, comme le principal agent de son entretien.

Or, comme le souligne V. A. Zelizer ${ }^{15}$, l'entrée de l'argent sur le territoire des dons n'est pas sans poser problème : comment les gens distinguent-ils les dons d'argent des autres transferts monétaires, alors que ce sont des billets physiquement identiques qui circulent ainsi ? Cette interrogation n'est-elle pas sous-jacente aux critiques des Maréens, qui estiment qu'à partir d'une certaine quantité d'argent en jeu, les dons monétaires perturbent «la coutume»? En fait, plusieurs techniques permettent aux Maréens de distinguer les dons monétaires des autres types de transactions. D'abord le mode de manipulation physique de l'argent: manière humble de l'offrir et de l'accepter, manière de recevoir, en tenant l'argent dans ses mains, à bout de bras, en le montrant à l'assistance ${ }^{16}$. Ensuite, les paroles qui accompagnent toujours ces dons d'argent: paroles de remerciements du donataire suivies d'un bref merci du donateur. Aux dons d'argent s'ajoutent toujours des «dons de parole». Enfin, l'argent est souvent l'objet d'un traitement physique particulier, il est comme embelli, apprêté : les billets sont enroulés ensemble et attachés
15. V. A. Zelizer, The Social..., op. cit., pp. 73, 82 et suiv.

16. Comme le souligne V. A. Zelizer, ibid., p. 109: «The physical manipulation of legal tender was a powerful, tangible technique of transforming money into a gift. » 


\section{O S S I E R}

Comment décrire les transactions Elsa Faugère Transactions monétaires en pays kanak
17. Alban Bensa, Jean Freyss, «La société kanake est-elle soluble dans l'argent?», Terrain, $\mathrm{n}^{\circ} 23$, 1994, p. 14. par des petites lianes, constituant des sortes de paquets cadeaux. Dans les mariages, lors de la présentation des dons, des morceaux de bois sont entaillés afin d'y glisser l'argent. Tous ces signes permettent à chacun de bien distinguer cet argent-là des autres formes d'argent qui ne sont pas l'objet de telles attentions.

V. A. Zelizer note qu'en plus des techniques de «marquage» physique des dons d'argent, il existe des restrictions quant à l'utilisation qui peut en être faite. Est-ce que les Maréens dépensent l'argent reçu dans les travaux coutumiers d'une manière particulière? Lorsque d'importantes sommes d'argent sont reçues, lors du mariage de sa fille par exemple, elles sont souvent utilisées pour des dépenses ostentatoires: achat d'une voiture ou construction d'une maison en dur. Mais de tels usages de l'argent ne paraissent pas spécifiques aux dons monétaires. Les fortes sommes d'argent gagnées d'un coup (grosse vente d'ignames par exemple), servent aussi à des dépenses ostentatoires - achat de mobilier, de maison, de voiture, etc. Les petites sommes d'argent, elles, sont réutilisées dans un autre travail coutumier, lorsque celui-ci est proche dans le temps. Cette réinjection ne se faisant cependant pas toujours avec les mêmes billets. Même si, dans les pratiques, les mêmes billets peuvent passer d'un domaine à l'autre, symboliquement, gains et dépenses domestiques restent distingués des gains et dépenses coutumiers.

Cette étude de cas montre bien que la présence de l'argent dans une cérémonie de fiançailles n'a pas transformé celle-ci en un espace marchand: Riri n'a pas «acheté» Élia. Pourtant les parents d'Élia peuvent utiliser l'argent reçu dans ce travail coutumier pour effectuer des transactions marchandes. Et c'est généralement ce qui se passe.

L'argent qui circule au sein de la parenté lors des cérémonies coutumières ne constitue pas un moyen de paiement. Il n'a pas la même signification que celui qui circule dans d'autres contextes, notamment marchands. Lors de ces cérémonies, l'argent qui passe de main en main, selon des réseaux de parenté très codifiés, ne sert pas à acquérir des marchandises. Il accompagne des paroles, des dons d'ignames, de viande ou de poisson, qui servent à matérialiser et à exposer publiquement les liens de parenté. Il n'est pas accumulé par l'un des protagonistes mais redistribué au sein de la parenté. Comme le note Alban Bensa $^{17}$ : 
«Les billets et les pièces émis par la Banque de France ont pris place dans les échanges kanaks: comme les $\hat{a} d i$, ils figurent dans les lots de biens identiques donnés et rendus, billets contre billets, pièces contre pièces, selon un mode de circulation qui annule la fonction même de la monnaie, son pouvoir de conversion et d'accumulation.»

Les $\hat{a} d i$, d'après A. Bensa, sont des objets précieux qui sont (ou étaient?) échangés entre eux, par les Kanaks du Centre-Nord de la Grande-Terre, lors de toute cérémonie importante ${ }^{18}$. Dans ce contexte, la signification de l'argent et son utilisation se rapprochent de celles qui étaient faites des richesses maréennes pendant la période précoloniale. Ce terme est utilisé par Marie-Joseph Dubois ${ }^{19}$ pour désigner les objets précieux (de pierre verte, de coquillage, etc.) qui étaient échangés, avant la colonisation, lors des événements sociaux importants. Cependant, si les richesses précoloniales ne permettaient pas d'acquérir d'autres types de biens mais circulaient uniquement dans des sphères d'échange de biens de prestige, l'argent permet d'acquérir n'importe quoi. Il permet de transgresser les différentes sphères d'échange, en passant de l'une à l'autre: la somme de 10000 FCFP reçue lors d'un mariage ou d'un deuil grâce à certaines logiques sociales, peut être convertie en marchandises. La surenchère monétaire présente dans les cérémonies coutumières transforme en partie les modalités d'acquisition du prestige. En effet, une certaine richesse financière permet à un clan de faire de grands «travaux coutumiers», privilège jusqu'alors réservé aux clans les plus peuplés et possédant un grand nombre de parents et d'alliés. Exposer publiquement ses réseaux de relations au cours des mariages et des deuils constitue autant un signe qu'un certain enjeu de prestige. Si la taille d'un clan et la quantité de ses alliés, dévoilées et mises à jour lors des cérémonies coutumières, sont source de prestige, la richesse financière peut, dans une certaine mesure, pallier une éventuelle faiblesse de telles relations sociales. Ce que les Maréens gardent en mémoire des cérémonies passées, ce sont justement les sommes monétaires qui étaient en jeu. Lorsque quelqu'un s'enquiert d'un mariage ou d'un deuil auquel il n'a pas assisté, sa première question est, systématiquement, «combien?»: «Combien le mort a-t-il fait?» ou «combien le mariage a-t-il fait?»

Les critiques des Maréens sur la monétarisation des coutumes peuvent ainsi s'interpréter comme le signe et le moyen de résister aux mutations sociales et économiques
18. Ibid. p. 14

19. Marie-Joseph Dubois, Maré, «Îles Loyauté. Le chemin des richesses», Journal de la Société des Océanistes, $\mathrm{n}^{\circ}$ 58-59, 1978, pp. 57-62. 


\section{O S S I E R}

Comment décrire les transactions Elsa Faugère Transactions monétaires en pays kanak
20. Voir K. R. Howe, Les îles Loyauté, Histoire des contacts culturels de 1840 à 1900, Société d'études historiques de la Nouvelle-Calédonie, Nouméa, 1978; Dorothy Shineberg, Ils étaient venus chercher du santal, Société d'études historiques de la Nouvelle-Calédonie, $n^{\circ} 3$, Nouméa, 1981.

21. F. Weber, Le travail à-côté, Paris, INRA-EHESS, 1989, pp. 173-192.

22. Jeanne Favret-Saada, Les mots, la mort, les sorts, Paris, Gallimard, 1977. en cours. On est dans une société hiérarchisée en fonction de sa naissance: on appartient à un clan prestigieux ou non, on est aîné ou cadet, homme ou femme, sujet ou Grand Chef. Cette transmission héréditaire du prestige et de la position sociale se trouve concurrencée par de nouvelles modalités d'acquisition du prestige et de la position sociale. N'importe qui - une femme, un jeune, un homme appartenant à un clan méprisé - peut gagner de l'argent. Dénigrer l'argent et valoriser «la vraie coutume» permet de maintenir l'ordre établi. Ce dénigrement de l'argent ne se produit pas seulement dans les transactions non marchandes. Il existe également dans les transactions marchandes où les créanciers sont bien plus stigmatisés que les débiteurs.

\section{Transactions marchandes et relations de parenté}

La participation des Maréens à une économie monétaire et marchande est attestée depuis les années $1870^{20}$. Selon K. R. Howe, dès cette époque, les Loyaltiens préféraient l'argent à tout autre article d'échange. Nombre d'entre eux partirent travailler sur la Grande-Terre, dans les mines de nickel et à Nouméa. Aujourd'hui encore, environ un tiers des Maréens vit en dehors de Maré. Pourtant, certaines transactions marchandes posent de véritables problèmes aux Maréens: celles qui ont lieu entre parents. Ces difficultés donnent lieu à d'incessantes accusations de «profitage». La question qui se pose est la suivante: à quelles conditions une transaction monétaire et marchande peut-elle avoir lieu entre parents?

\section{La faillite d'Albert}

Les Maréens se plaignent sans cesse des abus commis au nom de la coutume. D'après leurs discours, tout le monde utilise la coutume à tort et à travers pour prendre quelque chose (une poule, un sac de riz, un melon, une montre, de l'argent, etc.) à quelqu'un, notamment aux membres de sa parenté. Les accusations de «profitage» sont incessantes. Comment les interpréter? Comme les accusations de jalousie étudiées par F. Weber ${ }^{21}$ et celles de sorcellerie étudiées par Jeanne Favret-Saada ${ }^{22}$, elles ne sont jamais admises ni revendiquées par l'accusé. Personne ne dit jamais «je suis un profiteur». On peut également se demander, à la suite de F. Weber à propos des accusations de jalousie, si elles dénoncent des relations réelles ou imaginaires. Les Maréens profitent-ils effectivement des autres? 
L'utilisation de la coutume dans le but de capter une partie des ressources d'un parent s'effectue couramment au sein des espaces marchands que sont censés être les magasins tribaux. Il est d'usage d'y mettre en avant le lien de parenté avec le patron du magasin pour ne pas payer les marchandises ou pour différer le paiement c'est-à-dire obtenir un crédit. Les problèmes permanents de dettes non honorées sont à l'origine des échecs des nombreuses tentatives commerciales impulsées par des Maréens. Et il est extrêmement difficile de faire payer les débiteurs. À l'entrée des magasins, se trouve pourtant depuis quelques années une liste de noms avec le montant total des dettes contractées dans le magasin. Mais, manifestement, cet affichage public des dettes n'a guère d'effet. Il semble plus honteux pour le créancier de réclamer son dû ou de refuser de faire crédit que pour le débiteur d'être endetté, comme Albert en a fait la douloureuse expérience.

Albert a ouvert un magasin en tribu dans les années 1970. Âgé de 40 ans en 1996, il est très peu scolarisé et peu engagé politiquement. Les gens qui venaient dans son magasin, raconte-t-il, faisaient exprès de faire appel à la coutume pour profiter de lui et ne pas payer les marchandises. Il n'a pas réussi à leur refuser de faire crédit. $\mathrm{Au}$ bout du compte il a fait faillite et garde une amertume et une rancœur vivaces de ce qui s'est passé. Selon lui, les gens agissaient en toute connaissance de cause. Mettant en avant leur lien de parenté ( «je suis ton tonton, fais-moi crédit»...), ils cherchaient en fait à profiter de lui. En 1996, Albert envisage de monter un nouveau magasin. Il se sent prêt à affronter les problèmes de crédit qui entrâ̂nèrent sa première faillite. Plein de bonnes et fermes résolutions, il explique:

«Lorsque j'aurai le magasin, il n'y aura plus de copains! Copain copain comme ça. Mais en ce qui concerne le magasin, non! là c'est direct. Pas de crédit, rien du tout! Ah oui! Là, le jour où y'aura le magasin, y'aura plus de crédit !

- Tu avais eu des problèmes avec les crédits?

- Avec les crédits! Bouh! Jamais ils sont revenus! Ah! le crédit! il faut pas le dire, mais jusqu'au pasteur il déconne aussi! Ah oui! C'est pour ça, il faut avoir confiance en personne! J'ai l'amour, j'ai pas la haine mais je n'ai confiance en personne. J'ai confiance en personne. Même mon frère aussi, mon frère là, il est là, on est bien, mais j'ai pas confiance! Mon travail, c'est moi-même, hein! parce que le crédit ben y'en a, jusqu'à ce que le magasin ferme, mais le crédit c'est à bloc, mon cahier il est rempli, mais après je suis fatigué de marquer!

- Et les gens ils t'ont payé? 


\section{O S S I E R}

Comment décrire les transactions Elsa Faugère Transactions monétaires en pays kanak
23. «Hmihmi ni bo», termes nengone qui signifient «ton oncle maternel».
- Ils n'ont pas payé, beaucoup n’ont pas payé. Ici à Maré c'est comme ça, c'est... Parce qu'ici, il y a toujours la coutume et c'est là que tu t'embrouilles, avec la coutume. Par exemple si c'est tonton qui vient pour faire crédit, il dit: "Ah hmihmi ni $b o^{23}$, je suis ton tonton, alors fais-moi crédit!" Ah! mais tonton j'ai l'amour pour toi, mais il faut que tu penses au crédit! Il faut pas que tu dises tonton et après que tu partes, en disant c'est mon neveu! Ah bah! C'est ça la question, c'est toujours ça! » (extrait d'un entretien, janvier 1996).

Je lui demandais alors s'il avait parfois refusé de faire crédit. Il me répondit qu'il avait essayé, il avait même écrit sur la porte de son magasin qu'il ne faisait plus de crédit, mais les gens n'en tenaient pas compte et tentaient toujours leur chance. Face à leur insistance, il capitulait toujours ayant honte de leur refuser systématiquement un crédit. Il ajouta:

«C'est dur de sortir ici [s'élever socialement]. Il faut toujours, c'est un peu la coutume, la coutume c'est bon, mais là ils exagèrent avec la coutume. Ils font la coutume dans un magasin. Mais non c'est fini ça. Un magasin c'est pas la coutume. Mais ils font toujours comme une coutume mais ça ils ne comprennent pas, c'est un peu le mélange dedans.»

De telles pratiques ne mettent cependant pas un terme aux échanges «coutumiers». Ayant demandé à Albert s'il continuait, malgré tout, d'aider ses débiteurs lors des travaux coutumiers, il me répondit:

«Ah! oui oui! ça je vais toujours, je vais aider au travail, je suis obligé. Il ne faut pas dire que lui c'est personnel, y'a tout le monde derrière lui, toute sa famille. On ne peut pas ne pas aider, c'est obligé d'y aller toujours parce que là [le problème de crédit] c'est pas la question de toute sa famille, ça c'est personnel.»

D'après ces propos, les deux types d'échanges sont clairement distingués. Ce qui se joue dans un contexte marchand (dans son magasin) n'a pas de conséquence sur les échanges effectués lors des travaux coutumiers. Le nonremboursement des dettes engage la responsabilité du seul débiteur et non de l'ensemble de sa famille (ou de son clan), alors que les travaux coutumiers ne sont pas une affaire personnelle. Ils engagent la responsabilité collective (d'une famille et d'un clan) à laquelle il n'est pas possible de se soustraire. Refuser son aide à son débiteur lors d'un travail coutumier, engagerait, d'une part, la responsabilité de l'ensemble de son propre groupe de parenté et condamnerait, d'autre part, injustement l'ensemble du groupe de parenté de son débiteur puisque 
ce groupe n'est pas responsable des actes de chacun de ses membres.

L'existence, toujours actuelle, de ces problèmes de crédits révèle la relative impunité dont jouissent ceux qui commettent ces abus et qui entravent le «bon» fonctionnement de l'économie de marché. Ces pratiques paraissent ainsi peu condamnées et donc peu condamnables au regard des normes locales. La honte pèse, semble-t-il, davantage sur celui qui réclame son dû ou qui refuse tout crédit, que sur le débiteur.

\section{«Les profiteurs 》}

Le cas d'Albert montre l'existence "d'importantes forces de nivellement à l'intérieur des formes d'organisation locales, qui empêchent les efforts d'accumulation", pour reprendre les termes de P. Geschiere et de Peter Konings ${ }^{24}$. Pour bien comprendre ce qui se joue là et les raisons de ces «forces de nivellement», il faut analyser le double point de vue des «profiteurs» et de la «victime» de ces «profitages».

La règle voudrait que dans ces magasins s'effectuent des transactions marchandes. Or, nous dit cet homme, les clients, qui sont souvent des parents, tentent d'éviter ce type de transaction. Ils cherchent à transformer la transaction marchande en don, et ce, en jouant précisément sur leur lien de parenté. Se dévoile ici la difficulté d'instaurer et de faire respecter des transactions marchandes au sein de la parenté. L'utilisation de «la coutume » et du lien de parenté permet à ces «profiteurs» d'économiser. L'usage des normes coutumières dans l'espace marchand des commerces locaux tenus par des parents constitue une tactique aussi prisée qu'efficace. Pour bien comprendre ce type de pratiques, il ne faut pas perdre de vue que les Maréens et notamment ceux du Nord-Est de l'île où j'ai fait mes enquêtes de terrain sont bien souvent dans une situation où l'argent est rare. Seulement $6 \%$ de la population active de cette région étaient salariés au recensement de 1989 contre une moyenne de 29,3\% pour l'ensemble de l'île ${ }^{25}$. Leurs revenus monétaires proviennent principalement de la vente de leurs produits agricoles, des produits de la pêche et des aides sociales. Ce sont des revenus irréguliers et peu élevés. Pour ces Maréens, l'utilisation de la coutume dans la sphère marchande permet de compenser, en partie, la faiblesse de leurs ressources monétaires. L'enjeu n'est pas d'accumuler le maximum
24. P. Geschiere, Peter Konings (éd.), Itinéraires et modes d'accumulation au Cameroun, Paris-Leiden, Karthala et Afrika-Studiecentrum, 1993, introduction, pp. 9 et suiv.

25. Institut territorial de la statistique et des études économiques (ITSEE), 1989. 


\section{O S S I E R}

Comment décrire les transactions Elsa Faugère

Transactions monétaires en pays kanak

26. Comme le note

Jean-Pierre Olivier de Sardan dans Anthropologie et développement.

Essai en socio-anthropologie du changement social, Marseille, Paris, APAD-Karthala, 1995, p. 134 :

«La minimisation des risques est au cœur de nombreuses stratégies paysannes. »

27. Expression de Victor Turner (1920-1983). Grand anthropologue britannique, appartenant à la célèbre école de Manchester, il a travaillé en Rhodésie du Nord (actuelle Zambie), chez les Ndembu. Après avoir écrit un premier ouvrage centré sur les conflits ou «drames sociaux» (social dramas), Schism and Continuity in an African Society. A Study of Ndembu Village, Manchester, Manchester University Press, 1957, il poursuivit ses recherches en s'intéressant au phénomène rituel et à l'activité symbolique.

Parmi ses nombreuses publications, citons: Les tambours d'affliction. Analyse des rituels chez les Ndembu de Zambie, Paris, Gallimard, 1972;

Dramas, Fields and Metaphors. Symbolic Action in Human Society, Ithaca, New York, Cornell University Press, 1974.

28. F. Weber, «Transactions marchandes, ...», op. cit. d'argent, mais d'éviter de trop en dépenser. De telles stratégies économiques recherchent, avant tout, la sécurité. Ces stratégies de minimisation des dépenses et des risques transparaissent également dans les incessantes plaintes et critiques des Maréens sur la vie urbaine de Nouméa où tout est payant. Dans les cas où les revenus monétaires sont irréguliers, éviter, si possible, de dépenser de l'argent, permet de minimiser les risques ${ }^{26}$.

\section{Liens multiplexes ${ }^{27}$}

Pourquoi Albert n'a-t-il pas refusé aux membres de sa parenté de prendre des marchandises ou de leur faire crédit? On peut se demander comment nous-mêmes aurions réagi dans la même situation: aurions-nous refusé à notre mère un sac de riz ou un crédit si elle n'avait pas d'argent? La complexité du tissu social local où s'enchevêtrent différents types de liens sociaux fait qu'un client du magasin n'est pas seulement un client. Il est aussi un voisin, un frère, un cousin ou un oncle maternel. Au moment où la transaction marchande doit se produire, elle met chacun des partenaires de l'échange dans un rôle particulier, qui ne dure que le temps de la transaction: celui de vendeur et d'acheteur. Mais peut-on le temps de cette transaction faire abstraction des autres liens sociaux qui unissent ce vendeur et cet acheteur? À quelles conditions, finalement, une transaction monétaire et marchande apparaît-elle? D'après F. Weber, il y a «mise entre parenthèses des caractéristiques personnelles des partenaires de l'échange $\gg^{28}$. Or cette mise entre parenthèses n'est précisément pas effectuée ici. Bien au contraire, dans certains cas, les caractéristiques personnelles des partenaires empêchent l'instauration entre eux d'un échange marchand.

Pourtant, tous les Maréens ne sont pas de «mauvais payeurs». Et si les problèmes de crédit sont une difficulté bien réelle à laquelle se heurtent les magasins locaux, il n'en demeure pas moins que certains clients paient leurs marchandises, même à un membre de leur parenté. Est-ce toujours les mêmes individus qui ne jouent pas le jeu du marché? De quoi dépend l'instauration de transactions marchandes entre parents? Pourquoi certains jouent le jeu et d'autres pas? Est-ce lié au montant des revenus, à la nature du lien de parenté, au passif ou à l'historique de la relation, aux sentiments que l'acheteur éprouve vis-àvis du vendeur? 
La peur de la coutume: un écheveau d'accusations de jalousie, de «profitage» et de sorcellerie

Albert déclare que «c'est dur de sortir ici», c'est-àdire de s'élever socialement. Toutes sortes de discours mettent d'ailleurs en garde ceux qui auraient des velléités de «sortir du lot» et d'afficher leur réussite matérielle. Montrer des signes extérieurs de richesse expose, dit-on, à la jalousie qui risque fort de déclencher des attaques en sorcellerie. On entend souvent les Maréens dire: «la coutume c'est dangereux», «la coutume peut tuer »... Et, pour éviter de tels risques, disent-ils, il convient de bien respecter «la coutume», de «bien travailler», c'est-à-dire de bien redistribuer, de bien donner. Pour échapper aux dangers de la coutume, il faut donc en accepter les règles d'échange, de redistribution et de ponctions. Il ne faut pas exposer trop de signes extérieurs de richesse, ne pas trop montrer sa réussite matérielle ou bien en faire profiter les autres, sinon d'inévitables jalousies apparaîtront, causes, dit-on, de tous les «accidents», car «la coutume peut frapper» :

« Tu vois la coutume, il faut bien travailler, si tu travailles bien, c'est bon, la coutume elle veille sur toi, mais sinon, tu sais, c'est dangereux la coutume aussi! Elle peut te frapper la coutume. Par exemple, tu as mal quelque part, tu vas voir le docteur mais lui il ne peut pas te soigner, c'est la coutume qui t'a frappé, et seule la coutume peut te guérir sinon tu meurs! Il faut prendre des médicaments de la coutume. La coutume c'est dangereux, dangereux tu sais! Il faut faire attention. Et s'il y en a qui sont jaloux, alors là c'est pire, la coutume elle te frappe et tu meurs. On entend dire un tel il est mort de crise cardiaque, les vieux ils disent c'est le soleil, mais ils savent bien que ce n'est pas le soleil, il a été frappé par la coutume. C'est vraiment dangereux la coutume! C'est vraiment dangereux la coutume, ça peut faire le bien mais ça peut faire le mal aussi, faut faire attention» (un jeune homme de Hnadid, 29 ans, novembre 1995).

Cette peur de la «coutume» est la peur des «esprits», des «sorciers» et des sorts qu'ils peuvent jeter. Lorsque les Maréens évoquent leur peur de la coutume en disant qu'elle est dangereuse et qu'elle peut tuer, ils font plus particulièrement référence à ce qui les effraie le plus et qu'ils appellent le kaze. Un kaze est une sorte de paquet magique confectionné avec divers objets (os, plantes, pierre, etc.) et doté du pouvoir de donner la mort. Il se transmet au sein de la famille, mais il peut aussi s'acheter. Les kaze en provenance du Vanuatu sont réputés être extrêmement puissants et dangereux, à tel point que si 


\section{O S S I E R}

Comment décrire les transactions Elsa Faugère Transactions monétaires en pays kanak
29. Observation identique effectuée par A. Bensa pour les sociétés kanakes du Centre-Nord de la Grande-Terre: «ce rôle tout puissant attribué aux utérins se retrouve dans tout l'archipel» dans «Contraintes par corps et ordre politique dans les sociétés kanakes d'autrefois (Nouvelle-Calédonie) », version française d'un texte publié en anglais dans Oceania, décembre 1997, p. 20. l'on n'a pas transmis son kaze à quelqu'un, «on ne peut pas mourir», comme le dit une jeune femme:

«Il empêche que tu meurs, il te maintient en vie. Tu vois la vieille Bernadette, elle était déjà bien vieille. Et quand elle est partie à Houaïlou, elle a fait appeler ma grand-mère [pour lui donner le kaze]. Une heure après, elle était morte. Tu vois ce que je veux dire?»

Mais la peur de la coutume ne renvoie pas seulement à la peur des sorciers et des mauvais sorts qu'ils peuvent jeter. Les Maréens craignent tout particulièrement les malédictions provenant du clan des maternels. Comme l'expliquent tour à tour deux vieux hommes:

«Ils peuvent nous maudire. Il peut y avoir des malédictions parce que dans le sang de mes enfants, il y a aussi le sang de ma femme, c'est-à-dire de l'oncle maternel. Alors s'ils font quelque chose d'anormal, ce sang-là ça donne quelque chose de désastreux, une malédiction » (janvier 1996).

«L'oncle est sacré parce que c'est le frère de la mère. C'est l'oncle le plus sacré et s'il prononce une parole envers son neveu, là il y aura un pouvoir, ça va se réaliser parce que tonton ça veut dire sacré, c'est lui le frère de la mère. S'il se met en colère, tous ses neveux et nièces vont tomber dans le malheur parce qu'il a le sang de leur mère» (janvier 1996).

Même si la filiation est patrilinéaire, la parenté maternelle est très importante à Maré et notamment les liens entre neveux et oncles utérins. Le terme qui désigne l'oncle maternel (hmi) signifie également "prier, religion, sacré». Le sang, la chair et la vie se transmettent en ligne maternelle ${ }^{29}$. Un individu est socialement classé en fonction du groupe de parenté de son père (guhnameneng) mais également en fonction de ses parents maternels. On dit «qu'il sort de chez ses maternels, qu'il vient de chez eux» (puja $i$ ). L'expression puja $i$ est également utilisée pour les végétaux qui sortent de terre. La peur autour de la coutume tourne donc autour de la sorcellerie et de l'idée d'une «parenté sacrée» due à la transmission du sang.

\section{Une «exigence d'égalité» matérielle dans une société hiérarchisée et inégalitaire}

Cette peur de la coutume sert finalement à maintenir une certaine égalité matérielle ou, plutôt, les apparences de cette égalité. Les accusations de jalousie, qui sont au moins aussi fréquentes que celles de "profitage», «signalent la présence d'une égalité concurrentielle, c'est-à-dire d'une homologie de positions dans le 
groupe »30. À Maré, ces accusations tournent essentiellement autour de questions d'argent et de l'accès au salariat. Cette jalousie sociale s'est peut-être accentuée avec la politique de décentralisation impulsée par les accords de Matignon en 1988 qui a offert localement de nouvelles possibilités d'emplois salariés. En effet, l'objectif de «rééquilibrage» entre les zones rurales du Nord et des Îles, peuplées majoritairement de Kanaks et le Sud, davantage urbanisé et européanisé avec la ville de Nouméa, a ouvert aux Kanaks, pour la première fois dans l'histoire de la Nouvelle-Calédonie, l'accès à des emplois jusqu'alors réservés aux Européens.

Cette jalousie sociale révèle une «exigence d'égalité » matérielle, alors même que la société kanake est très inégalitaire et très hiérarchisée ${ }^{31}$. Certains Maréens appartiennent à des clans prestigieux (plus anciens, ils détiennent des propriétés foncières), d'autres à des clans méprisés ${ }^{32}$. Un clan est lui-même hiérarchisé selon l'ordre de naissance: d'abord l'aîné du lignage aîné, puis tous ceux de ce même lignage par ordre de naissance; puis l'aîné du lignage cadet, et tous ceux de ce même lignage par ordre de naissance, etc. Le premier né du lignage aîné est généralement le chef du clan. De plus les désaccords sur les généalogies sont permanents.

Ainsi, Albert appartient à un clan méprisé parce qu'il n'est arrivé à Maré qu'à la fin du XIX ${ }^{\mathrm{e}}$ siècle. On dit que ses membres fuyaient alors l'île voisine d'où ils avaient été chassés. Son appartenance clanique peu glorieuse et son absence d'assise foncière ont joué en défaveur de son entreprise commerciale. Il était d'autant plus facile à ceux qui le souhaitaient, de profiter de lui dans son magasin, que sa position était fragile. Certains pouvaient, par exemple, le menacer de reprendre la terre sur laquelle son magasin était construit, les revendications foncières des terres sur lesquelles quelqu'un gagne de l'argent étant très fréquentes.

\section{Le pentecôtisme: un moyen de "s'en sortir»?}

La marge de manœuvre dont dispose Albert est donc étroite. L'un des moyens pour «s'en sortir» localement est la conversion au pentecôtisme, ce qu'il a fait en 1993. Ce culte permet en effet de légitimer, aux yeux des convertis, le non respect des règles de «la coutume» et de la parenté. Lors de leur implantation à Maré, dans les années 1980, les pasteurs pentecôtistes s'opposaient à «la coutume», et même si aujourd'hui, ils ont assoupli leur
30. F. Weber, Le travail..., op. cit., p. 176.

31. Pour une description détaillée de l'organisation sociale et coutumière des chefferies et des clans de Maré, voir M. J. Dubois, «Les chefferies de Maré. Nouvelle-Calédonie», thèse de doctorat, université de Paris V, Lille, 1977; Mythes et traditions de Maré, Nouvelle-Calédonie, Les Eletok, Publications de la société des Océanistes, n 35, Paris, 1975. Voir aussi Elsa Faugère, «L'argent et la coutume... », op. cit., pp. 75 et 86-121. Pour une description de l'organisation sociale des sociétés kanakes du Centre nord-est de la Grande-terre, voir A. Bensa et Jean-Claude Rivierre, Les chemins de l'alliance. L'organisation sociale et ses représentations en NouvelleCalédonie, Paris, SELAF, 1982.

32. Il serait trop long d'expliquer ici en détail le fonctionnement de ce que les Maréens appellent «clans». Le terme nengone est guhnameneng qui, littéralement, signifie «l'endroit où l'on reste». Pour des raisons de commodité, j'emploie ici le terme de «lignage» sans entrer dans la complexité terminologique de ce terme très controversé et très discuté dans l'histoire de l'anthropologie, anglo-saxonne et française notamment. 


\section{O S S I E R}

Comment décrire les transactions Elsa Faugère Transactions monétaires en pays kanak
33. Le terme de «boucan» désigne les mauvais esprits et les mauvais sorts qui peuvent être jetés.

C'est la traduction française courante du terme kaze. position, ils continuent d'adopter une position critique et de rester à l'écart de la vie locale. Les autres confessions, protestante (Église libre et Église évangélique) et catholique, implantées depuis longtemps, ont intégré la coutume à tel point qu'il est impossible de faire la part entre les deux. Le pentecôtisme, en revanche, constitue une arme contre la coutume, un moyen d'échapper à la peur qu'elle provoque parce qu'il dénie toute réalité à l'existence des pratiques de sorcellerie, et place les adeptes sous la puissante protection de Jésus. Il forme ainsi une sorte de refuge et de rempart contre toute attaque en sorcellerie, contre tout mauvais sort, comme l'explique un jeune de Wakone, converti depuis 1993:

«Maintenant, avec Jésus mais je n'ai plus peur. Je marche la nuit sur la route mais j'ai pas peur des boucans ${ }^{33}$. Mais, avant, quand je devais marcher depuis le temple jusqu'ici mais je courais! Là, non, parce que j'ai Jésus et il est plus fort qu'eux. Si jamais il y a quelqu'un qui a un boucan pour faire du mal mais seulement en posant ma main dessus je peux le faire sortir» (décembre 1995).

Il ajouta que son frère (qui n'est pas pentecôtiste) a toujours un «protège» sur lui autour du poignet, acheté à un homme de Penelo.

«Dessus il y a des médicaments. Parfois ils mettent aussi un foulard sur la tête avec des trucs dessus pour se protéger. [...] Parce qu'ici, poursuit-il, il y a beaucoup de jeunes et de vieux qui font les boucans. L'autre soir, pendant un mariage dans une autre tribu, un jeune d'ici a été emboucanné par un mec de làbas. Il est tombé par terre mais heureusement il y avait quelqu'un qui avait un protège et qui lui a frotté le visage avec, comme ça le boucan est sorti. Il y a plein de boucans ici. Ceux qui n'y croient pas, ils ne risquent rien, ils ne peuvent pas être touchés par les boucans. Moi j'ai pas peur parce que j'ai Jésus.»

Réputé efficace, le pentecôtisme n'est qu'une parade. Des solutions d'un ordre tout différent permettent d'établir des transactions marchandes entre parents: la coopérative commerciale et le marché municipal. Elles font toutes deux intervenir un collectif d'une autre nature que les collectifs de parenté.

\section{Les coopératives et le marché municipal}

Patrick est un des premiers et rares ingénieurs agronomes kanaks. Il a fait ses études supérieures en métropole dans le cadre de l'«Opération 400 Cadres». Cet ambitieux programme de formation lancé par les accords de Matignon avait comme objectif de former quatre cents 
cadres kanaks. Patrick est engagé politiquement dans des mouvements indépendantistes. Selon lui, ces problèmes de dettes non honorées s'expliquent culturellement:

«Nous, les Kanaks, on ne sait pas trop gérer l'argent européen. Avant, il y avait les monnaies traditionnelles. C'est nous qui les faisions tandis que pour gagner l'argent européen, il faut suer! Tous les premiers magasins des Kanaks, ils se sont cassés la gueule parce que si quelqu'un venait acheter du sucre et qu'il lui manquait 50 ou $100 \mathrm{~F}$ et que c'était un cousin... le vieux il ne pouvait pas dire "non tu prends pas le sucre parce que tu n'as pas assez d'argent!" Alors il prenait le sucre et puis à la fin il n'y avait pas d'argent dans la caisse. On dit que c'est la coutume, mais Ballande ${ }^{34}$ et les autres, ils font pas la coutume! Alors, au contact des Européens, on a appris à mieux gérer l'argent. C'était dans les années 1960, avec les coopératives, parce que comme c'était en commun, si la famille venait, on ne pouvait pas leur dire prends même si t'as pas d'argent, parce qu'il disait c'est pas à moi le magasin, c'est à plusieurs. C'était une manière un peu détournée, comme ça c'était possible» (octobre 1995).

En effet, une des solutions pour pouvoir effectuer des transactions marchandes entre parents est de se mettre en coopérative. Le magasin et ses marchandises devenant propriété collective, le vendeur peut refuser, précisément au nom de ce collectif, que le «client-parent» ou le «parent-client» ne prenne une marchandise sans payer. Parce que les marchandises n'appartiennent plus seulement au vendeur mais à un groupe, le lien de parenté ne permet plus de prendre un produit sans payer. L'appartenance à ce collectif permet de rompre le lien dyadique de parenté. En invoquant une propriété commune des marchandises, il devient possible de refuser un don à un parent, d'instaurer avec lui une transaction marchande.

Le recours au collectif est également la solution utilisée au marché municipal de La Roche. Dans la région nord-est de Maré, ce marché représente la principale filière de commercialisation des produits de l'agriculture, de la pêche et de la chasse. Il a été construit en 1990 en remplacement d'un ancien marché qui datait des années 1970. Jusqu'à la construction du nouveau marché, les produits n'avaient pas de prix au kilogramme. Chacun vendait au prix qu'il fixait lui-même. La gestion du marché et la fixation des prix de tous les produits qui y sont vendus font aujourd'hui l'objet d'un travail collectif. L'ensemble des vendeurs forme ce qu'on appelle le «bureau du marché» qui détermine les prix des produits.
34. Nom d'une famille de colons bordelais venu au $\mathrm{XIX}^{\mathrm{e}}$ siècle en Nouvelle-Calédonie où ils firent fortune grâce à des activités d'import-export. 


\section{O S S I E R}

Comment décrire les transactions Elsa Faugère

Transactions monétaires en pays kanak

35. Voir, notamment, Isabelle Merle, Expériences coloniales. La NouvelleCalédonie (1853-1920), Belin, Paris, 1995; A. Bensa, Chroniques Kanak, l'ethnologie en marche, Ethnies, $\mathrm{n}^{\circ}$ 18-19, 1995; A. Bensa, Nouvelle-Calédonie. Vers l'émancipation, Paris, Gallimard, 1998; John Connell, «New Caledonia: Social Change, Political Change and Tradition in a Settler Colony ", in A. B. Robillard, (éd.), Social Change in the Pacific Islands, Londres et New York, Kegan Paul International, 1992, pp. 65-97: J. Connell, New Caledonia or Kanaky? The political History of a French Colony, Canberra, The Australian National University, Pacific Research Monograph, $n^{\circ} 16$, 1987; Stephen Henningham, France and the South Pacific. A Contemporary History, South Sea Books, Honolulu, University of Hawaii Press, 1992. Voir aussi J. Freyss, Économie assistée et changement social en NouvelleCalédonie, Paris, IEDES, 1995, dont les deux premières parties qui dissèquent les fondements de l'économie néocalédonienne sont passionnantes.
Lorsqu'on entre dans la halle, un grand tableau noir domine l'espace de vente avec, écrits à la craie, les prix de chaque produit. Il faut en effet se réunir pour déterminer, ensemble, les prix. Cette fixation collective des prix permet un contrôle du groupe, mais elle réduit la marge de manœuvre des vendeurs, limitant du même coup les pressions potentielles des acheteurs.

$$
\text { * } *
$$

Confondre argent et marché, transaction monétaire et transaction marchande, conduit à mal penser et l'argent et le marché. Donner de l'argent pour demander la main d'une femme ne signifie pas qu'on l'achète. Et acheter un kilo de légumes à sa cousine au marché municipal ne signifie pas que la transaction est devenue impersonnelle. Cette confusion empêche d'analyser correctement les interactions à l'échelle micro-sociologique. Mais elle empêche également de comprendre et d'analyser la situation macro-économique et notamment les raisons pour lesquelles les Kanaks n'occupent qu'à la marge des positions économiques dominantes. S'ils sont économiquement dominés, ce n'est pas à cause d'une coutume qui serait un frein au développement. La coutume a bon dos. Prétendre que, à cause d'elle, les Kanaks sont marginalisés ou dominés économiquement conduit à ne pas s'interroger sur les fondements d'une économie néocalédonienne responsable de cette situation ${ }^{35}$. 